\title{
Gas-dynamic density downramp injection in a beam-driven plasma wakefield accelerator
}

\author{
J. P. Couperus Cabadağ $\odot,{ }^{1,}{ }^{*}$ R. Pausch, ${ }^{1}$ S. Schöbel, ${ }^{1,2}$ M. Bussmann,,${ }^{3,1}$ Y.-Y. Chang, ${ }^{1}$ S. Corde, ${ }^{4}$ A. Debus, ${ }^{1}$ H. Ding,,${ }^{5,6}$ \\ A. Döpp,${ }^{5,6}$ F. M. Foerster, ${ }^{5}$ M. Gilljohann, ${ }^{4,5,6}$ F. Haberstroh, ${ }^{5}$ T. Heinemann, ${ }^{7,8,9}$ B. Hidding, ${ }^{8,9}$ S. Karsch, ${ }^{5,6}$ A. Koehler, ${ }^{1}$ \\ O. Kononenko, ${ }^{4}$ A. Knetsch, ${ }^{4}$ T. Kurz, ${ }^{1,2}$ A. Martinez de la Ossa, ${ }^{7}$ A. Nutter, ${ }^{8,9}$ G. Raj, ${ }^{4}$ K. Steiniger, ${ }^{1}$ U. Schramm, ${ }^{1,2}$ \\ P. Ufer, ${ }^{1,2}$ and A. Irman ${ }^{1}$ \\ ${ }^{1}$ Helmholtz-Zentrum Dresden - Rossendorf, Institute of Radiation Physics, Bautzner Landstrasse 400, 01328 Dresden, Germany \\ ${ }^{2}$ Technische Universität Dresden, Faculty of Physics, 01069 Dresden, Germany \\ ${ }^{3}$ Center for Advanced Systems Understanding, Untermarkt 20, Görlitz, Germany \\ ${ }^{4}$ LOA, ENSTA Paris, CNRS, Ecole Polytechnique, Institut Polytechnique de Paris, 91762 Palaiseau, France \\ ${ }^{5}$ Ludwig-Maximilians-Universität München, Faculty of Physics, Am Coulombwall 1, 85748 Garching, Germany \\ ${ }^{6}$ Max Planck Institut für Quantenoptik, Hans-Kopfermann-Str. 1, Garching 85748, Germany \\ ${ }^{7}$ Deutsches Elektronen-Synchrotron DESY, Notkestraße 85, 22607 Hamburg, Germany \\ ${ }^{8}$ The Cockcroft Institute, Keckwick Lane, Warrington WA4 4AD, United Kingdom \\ ${ }^{9}$ University of Strathclyde, 107 Rottenrow, Glasgow G4 ONG, United Kingdom
}

(Received 13 April 2021; revised 2 July 2021; accepted 13 September 2021; published 15 October 2021)

\begin{abstract}
We present the experimental demonstration of density downramp injection at a gas-dynamic shock in a beamdriven plasma accelerator. The ultrashort driver electron beam with a peak-current exceeding $10 \mathrm{kA}$ allows operation in the blowout regime and enables injection of electron witness bunches at gentle density ramps, i.e., longer than the plasma wavelength, which nurtures prospects for ultralow bunch emittance. By precision control over the position of injection we show that these bunches can be energy-tuned in acceleration gradients of near $120 \mathrm{GV} \mathrm{m}^{-1}$.
\end{abstract}

DOI: 10.1103/PhysRevResearch.3.L042005

Beam-driven Plasma Wakefield Accelerators (PWFAs) enable dephasing-free acceleration of electron bunches at gradients $>100 \mathrm{GV} \mathrm{m}^{-1}$ when a plasma wakefield is excited by a relativistic particle beam of high peak-current [1,2]. Operation of a PWFA in the blowout regime [3,4] results in stable wakefield formation with linear uniform focusing fields, a prerequisite to enable emittance preservation during acceleration. Rapid progress was made in recent years with the demonstration of energy doubling of $42 \mathrm{GeV}$ electrons [5], of high energy-transfer efficiency from driver to witness beam [6], and with the proof-of-concept addition of plasma photocathodes for witness beam injection [7]. Development of internal injection methods in PWFAs is a crucial task toward high-quality low-emittance bunch generation and improved control over bunch parameters for quality-demanding applications like free-electron lasers [8] or high energy colliders [9].

Equally vital as bunch quality for applications is the ability to control and adjust bunch parameters. The injection mechanism plays a pivotal role in both aspects, as it determines the initial properties a witness bunch acquires in the wakefield, including its emittance. Additionally, if able to control and adjust the point of injection within a PWFA, independent of

\footnotetext{
*j.couperus@hzdr.de

Published by the American Physical Society under the terms of the Creative Commons Attribution 4.0 International license. Further distribution of this work must maintain attribution to the author(s) and the published article's title, journal citation, and DOI.
}

other accelerator parameters, one achieves on-demand tunability of the final energy. In recent years, several injection methods directly inside a PWFA have been proposed aiming for the generation of ultralow bunch emittance [10-16]. Injection triggered by a plasma density downramp [10,17] is especially appealing for its adjustability and its potential for witness injection with only few tens of nanometer emittance [18,19].

In this letter, we present the first experimental implementation of such a density downramp injection (DDI) scheme induced by a hydrodynamic shock-front in a PWFA. We show energy tuning capability by means of tuning the downramp location in a high-density, high accelerating gradient PWFA. Downramp injection can only occur when the longitudinal velocity of plasma electrons $\beta_{z}$ temporally exceeds the wake's phase velocity $\beta_{\phi}$, i.e., $\beta_{z}>\beta_{\phi} \approx\left(1+\frac{\chi}{2 k_{p} n_{p}} \frac{\mathrm{d} n_{p}}{\mathrm{~d} z}\right)^{-1}$, which reduces while the driver traverses a negative density gradient. Here $n_{p}(z)$ is the local plasma density, $\chi=k_{p}\left(z-\beta_{b} c t\right)$ the electron's phase relative to the driver and $\beta_{b}$ the driver speed relative to the speed of light. For this condition to be fulfilled, the density gradient has to be sufficiently steep and the driver has to excite a strong enough blowout to generate sufficiently fast wake electrons, which typically only occurs when the drive beam peak-current exceeds a few kilo-amperes. Generally, stronger drivers lead to a higher longitudinal velocity of plasma electrons when returning to the propagation axis $\left(\beta_{z}\right)$ and thus allow for injection to occur at more gradual density ramps [17]. Despite being a common injection scheme in laser-driven wakefield accelerators (LWFAs) [20-25], fulfilling both requirements simultaneously in linac-driven PWFA 

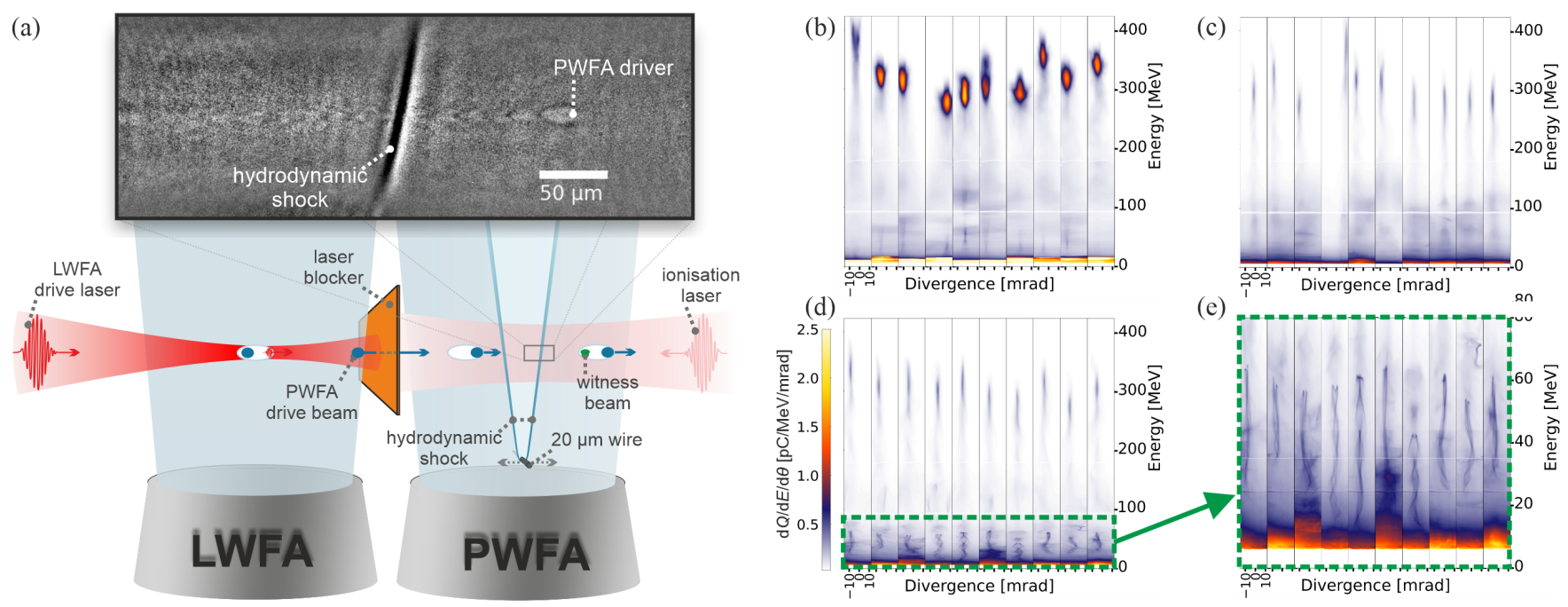

FIG. 1. (a) Experiment schematic. Two jets form separated accelerators: Laser driven (LWFA, left) and beam driven (PWFA, right). From left to right, a high-intensity laser pulse drives the LWFA, where the PWFA drive beam is generated. A kapton foil reflects the spent LWFA drive laser, while the electron beam propagates into the PWFA as a drive beam. A low-intensity counterpropagating laser preionizes the PWFA medium. Optionally, a position-adjustable $20-\mu \mathrm{m}$ wire can be inserted inside the PWFA stage to invoke a hydrodynamic shock-front. The density downramp of this shock allows for injection of a witness bunch at a predetermined position. The inset shows a shadowgram of the beam-driven plasma wave at the second shock-front captured by a few-fs probe beam. Panels (b)-(d) each display 10 consecutive electron distributions [all using the color-scale shown in (d)] used for determining the statistical parameters. Panel (b) shows the drive beams before interaction with the PWFA stage. They are transmitted through the blocker foil while the PWFA is still disabled. Panel (c) shows the remaining drive beams after interaction with the PWFA stage enabled but no shock-wire present. Panel (d) shows the spectra with wire for DDI at the PWFA center. Accelerated witnesses are visible at low energies, highlighted in (e) as a zoom view of (d).

facilities [26,27] is especially challenging at shallow ramps [10] linked to the relatively low $\beta_{z}$ attainable with currently available PWFA drive beams [17]. At steep ramps (downramp length $l$ shorter than $c / \omega_{p}$ ) DDI has recently been demonstrated $[7,28,29]$ using a laser-generated cold plasma torch [11], which offers particularly steep density gradients. In contrast, the gas-dynamic shock injection regime produces gentle density downramps (i.e., $l>c / \omega_{p}[19]$ ), which promises a more adiabatic injection process with particularly low emittance for the injected witness bunch [18].

In our experiment, shown in Fig. 1(a), we generate the PWFA drive beam in a preceding laser-driven LWFA. This staged LWFA-driven PWFA (LPWFA) [30-32] exploits the high peak-currents from the LWFA beam, which exceed 10 kiloamperes [33,34], to drive the PWFA such that the injection requirements are met even on gentle gas density downramps. Meanwhile, the inherent ultrashort electron beam duration of around $10 \mathrm{fs}$ [35] allows operation at plasma densities above $10^{18} \mathrm{~cm}^{-3}$. These high plasma densities lead to high amplitude wakefields and the potential for high-brightness witness generation [36].

Our setup consists of two consecutive gas jets forming the two separate laser- and beam-driven accelerators. The 3-mm-long LWFA is driven by the 150 TW DRACO laser [37] and is operated in a tailored self-truncated ionization-induced injection (STII) scheme detailed in Ref. [38] and employs beam loading to limit energy spread [33]. The generated beam exhibits a broad energy spectrum with a distinguishable quasimonoenergetic fraction which contains a mean high-charge $Q$ of $335 \pm 24 \mathrm{pC}$ within its FHWM and $39 \pm 2 \mathrm{MeV}$ FWHM energy spread at a mean energy within the FWHM,
E, of $323 \pm 9 \mathrm{MeV}$ (S.E.M.). This corresponds to a spectral charge density $S=Q /(\Delta E / E)$ of $28 \pm 2 \mathrm{pC}^{-1}$. The beam duration (FWHM) was reconstructed at an equivalent configuration from the spectral intensity of transition radiation measurements $[39,40]$ to $14.8 \pm 1.6 \mathrm{fs}$ at an envelope peak-current of $12.4 \pm 1.1 \mathrm{kA}$ [41], sufficient to drive the subsequent PWFA in the strong blowout regime. A $25 \mu \mathrm{m}$ kapton foil placed in vacuum, $0.5 \mathrm{~mm}$ behind the LWFA, acts as a plasma mirror to fully reflect the spent drive laser. Before entering the PWFA as a drive beam, the beam divergence was measured to be $3.1 \pm 0.2 \mathrm{mrad}$ (rms). This comparably high divergence results from current filamentation instability created by high-intensity laser-foil interaction before foil transition [42]. The spectral distributions of a set of consecutive drive beams is shown in Fig. 1(b). Throughout this letter the operational conditions of the LWFA are kept constant at the aforementioned conditions.

The PWFA consists of a 3-mm hydrogen jet, placed $0.5 \mathrm{~mm}$ behind the blocker foil. Using a counterpropagating low-power $(\sim 20 \mathrm{~mJ}$ with a FWHM focal spot size of $\sim 120 \mu \mathrm{m}$ ) laser pulse, which leaves the gas jet through the left edge about $1 \mathrm{ps}$ before the drive electron beam enters the gas jet through the left edge, a preformed plasma channel defines the PWFA medium. The PWFA is first operated without density downramp, i.e., no shock wire inserted. At a plasma density of $4.0 \times 10^{18} \mathrm{~cm}^{-3}$, a strong beam-plasma interaction is observed in the remaining driver spectra, as shown in Fig. 1(c). The driver's peak FWHM-integrated charge is reduced to an average of $50 \pm 4 \mathrm{pC}$. The energy spread broadens to $69 \pm 9 \mathrm{MeV}$, while the associated mean peak energy reduces to $302 \pm 10 \mathrm{MeV}$. The spectral charge density 


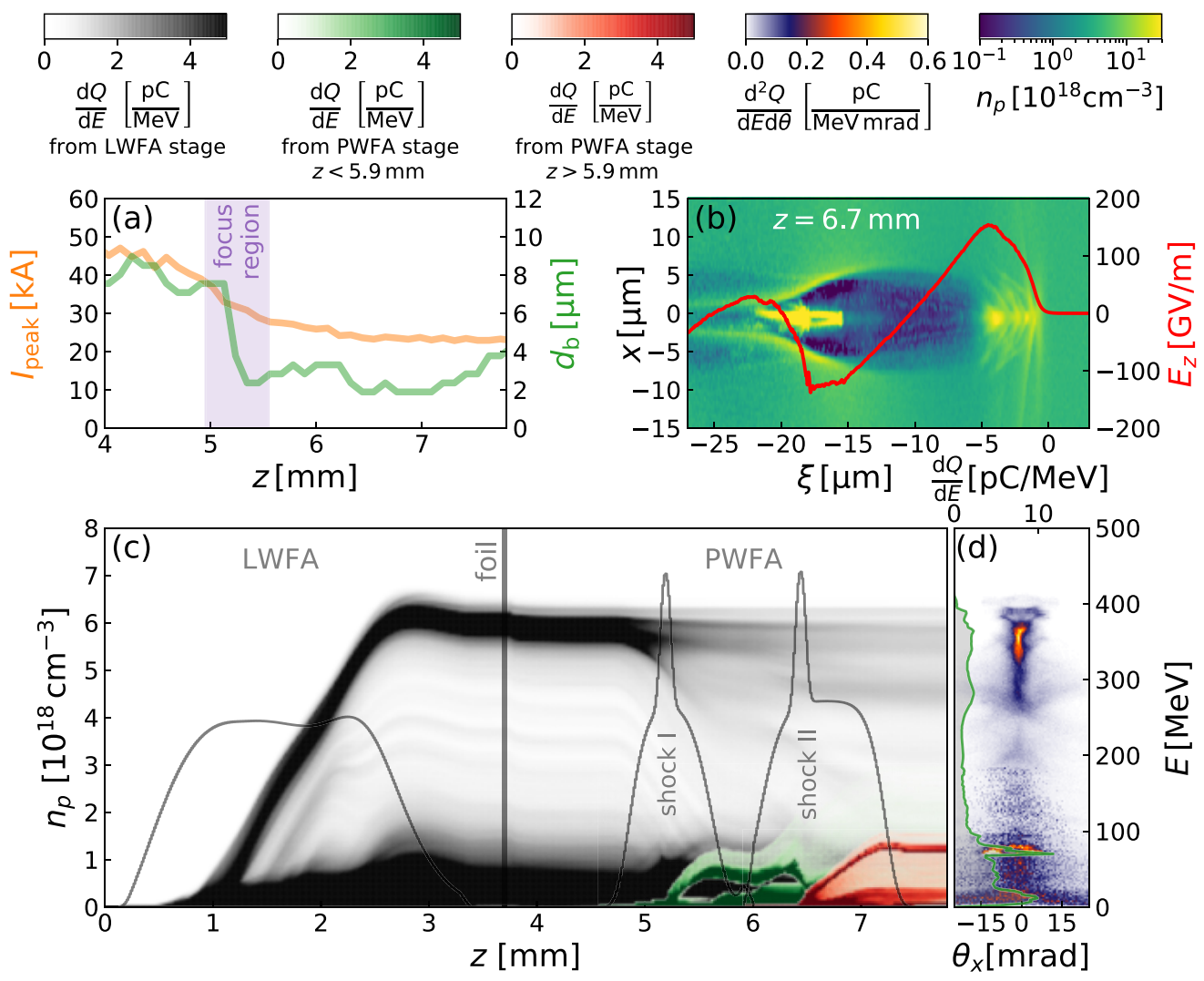

FIG. 2. LPWFA simulation with shocks induced by a wire in the center of the PWFA stage: Panel (a) depicts the evolution of the driver current (orange) and diameter (FWHM, green) over the longitudinal position $z$. Panel (b) shows a density snapshot after the second shock illustrating an accelerating gradient of $130 \mathrm{GV} \mathrm{m}^{-1}$. Panel (c) shows the energy evolution $E$, with gray, green, and red presenting, respectively, electrons originating in the LWFA stage and the PWFA stage before and after the wire alongside the density profile $n_{p}$. The loss of the first shock injection and the acceleration of the second shock injection is visible in (c) at $z \approx 6.5 \mathrm{~mm}$. Panel (d) shows the final electron energy distribution on a synthetic spectrometer.

is reduced to $2.3 \pm 0.2 \mathrm{pC} \%$. These effects are signatures of energy transfer from the drive beam into the plasma for the excitation of a plasma wakefield. However, as confirmed in simulations, a fraction of the driver beam is decelerated to energies significantly below the FWHM lower bound, thus these values do not contain the entire driver beam and do not allow us to determine exact charge loss and energy transfer values from experimental measurements. Shadowgraphy probe images show wakefield formation by revealing a distinct plasma wave captured by a synchronized few-cycle laser pulse [43]. The presence of these wakefields together with a reduction by a factor of more than two in divergence, i.e., down to $1.4 \pm 0.1$ mrad, demonstrates that a substantial fraction of the drive beam originating from the LWFA is successfully transported and refocused by utilizing the initial part of the hydrogen jet as a passive plasma lens [44-47]. Due to the lack of an injection mechanism at this configuration, no witness bunch is observed in the spectra shown in Fig. 1(c).

To prompt injection of a witness, we introduce a density downramp by placing a $20-\mu \mathrm{m}$ wire $2 \mathrm{~mm}$ below the propagation axis of the driver, down from the center of the PWFA. This induces a hydrodynamic shock and creates two distinct density downramp regions [Fig. 2(c)]. The gas-flow disruption is highly localized to prevent significant influencing the
PWFA gas jet upramp required to recapture the drive beam. As we will show in more detail later, electrons injected at the first shock are fully decelerated while passing through the second shock. Therefore, despite two shocks, a single witness bunch is expected in this arrangement. Exiting the PWFA, the remaining driver shows similar beam parameters as in the case without wire; a reduced mean divergence at $1.5 \pm 0.1 \mathrm{mrad}$, a likewise reduced peak charge at $55 \pm 4 \mathrm{pC}$, a mean peak energy of $301 \pm 7 \mathrm{MeV}$ with $71 \pm 5 \mathrm{MeV}$ energy spread, and a spectral charge density of $2.4 \pm 0.1 \mathrm{pC} \%^{-1}$. Since a similar response is found as in the case without shock wire, we deduce that the drive beam is sufficiently rigid not to be disturbed by the induced plasma density steps. With the introduction of a DDI region, the emergence of witness bunches accelerated up to a mean maximum energy of $57 \pm 2 \mathrm{MeV}$ is observed as shown in Fig. 1(d). This value is determined at the most energetic witness signature, prominently distinguished from the reference average without shock, and averaged over all events showing injection. Furthermore, the large energy spread down to few-MeV energies indicates that witness electrons are injected at different phases of the wakefield. This can be attributed to a large density step from the shock-peak down to the plateau region leading to the injection of long witness bunches [17]. Here, the bunch experiences a high 
accelerating gradient difference between the head, closer to the accelerating field's zero crossing point, and the tail, at the rear-side of the wakefield.

To assure the origin of the injection, a 3D particle-in-cell (PIC) simulation is performed using PIConGPU [48] implementing a LWFA-PWFA digital twin of the experiment. We achieve start-to-end simulation capability by integrating all stages of the experiment into a single, large-scale, GPUaccelerated simulation on 192 K80 GPUs, mimicking the experiment as closely as possible while ensuring numerical stability for more than $3.6 \times 10^{5}$ PIC cycles.

The simulation models the LWFA drive laser using a Gauss-Laguerre mode, up to order 12, fitted to the measured laser profile within one Rayleigh length around the focus. The gas profile is based on measurements using tomographic interferometry [49] which was further refined by plasma-wave shadowgrams. The latter provides higher spatial resolution for the determination of the exact position of the highly localized shock-fronts. However, the simulated shock density profile is still an approximation, which may result in deviations in witness quality compared to experiment. Inserting a wire perturbs the gas, creating two shock regions to the left (shock I) and right (shock II) of the wire with reduced density in its shadow [Fig. 2(c)].

The simulation box consists of $768 \times 4608 \times 768$ cells with a transversal resolution of $177.2 \mathrm{~nm}$ and a longitudinal resolution of $22.15 \mathrm{~nm}$. This resolution ensures numerical stability throughout this start-to-end LPWFA. A combined BSI [50] and ADK [51] ionisation model was used with the Lehe [52] field solver, the Boris pusher [53], and the Esirekepov current deposition scheme [54]. A full setup of the simulation can be found under [55]. The beam's transition through the foil is simplified, due to including this being prohibitively expensive computationally, thus neglecting the complex dynamics resulting from the interaction between the magnetic instabilities at the surface of the foil and the electron bunch and its influence on the divergence during transition [42].

With a charge of $226 \mathrm{pC}$, a divergence of $3.4 \mathrm{mrad}(\mathrm{rms})$, and an FWHM energy spread of $30 \mathrm{MeV}$ around a mean peak energy of $366 \mathrm{MeV}$ after passing through the foil, the simulated driver approximates the parameters of the experiment. After expansion in the intermediate vacuum, the drive beam is effectively recaptured as it enters the PWFA stage. Most of the beam is focused down to a FWHM beam diameter of $2.4 \mu \mathrm{m}$ [Fig. 2(a)] while a substantial portion, mostly consisting of its higher divergence fraction, is lost and does not contribute to the wakefield formation. A high driver density, much larger than the ambient plasma density $\left(n_{\mathrm{b}} \gg n_{\mathrm{e}}\right)$, with a peak-current $I_{\text {peak }}>10 \mathrm{kA}$ is achieved and sustained during propagation while the short beam duration condition remains fulfilled. This enables the driver to excite a strong enough blowout, providing fast electrons required to trigger shockfront injection and subsequently accelerate witness electrons, as illustrated in Fig. 2(b).

The simulation further reveals that the density downramps at both shock-fronts lead to injection, i.e., green and red shades illustrate electrons from shocks I and II, respectively [Fig. 2(c)]. However, only the witness electrons from the second shock stay in phase with the accelerating field and continuously gain energy. After injection at $z_{\mathrm{i}}$, the relative phase $\chi(z)$ of an electron within the wake changes with the density $n_{p}(z)$ as $\chi(z)=\chi\left(z_{\mathrm{i}}\right) \sqrt{n_{p}\left(z_{\mathrm{i}}\right) / n_{p}(z)}$. Electrons only experience acceleration in the back half of the cavity at $-2 \pi \lesssim$ $\chi \lesssim-\pi$ [17]. The witness injected at shock I is generated in the low-density shadow region of the wire and rephases to a decelerating region of the second cavity $(\chi \lesssim-2 \pi)$ during the transition through shock II, where it decelerates quickly. In contrast, the witness bunch generated at shock II is injected spatially separated and does not undergo further density steps and is accelerated over the remaining length of the accelerator with an average accelerating gradient of $96 \mathrm{GVm}^{-1}$ reaching $74 \mathrm{MeV}$ shown in Fig. 2(d).

Experimentally, taking advantage of the localized injection, we achieve energy tuning capability by adjusting the position of injection and thus changing the effective acceleration length of the injected witness. Figure 3 presents the witness energy as a function of shock II position. The energy gain is directly related to the change of the acceleration length, which allows us to determine the effective accelerating gradient to $85 \mathrm{GV} \mathrm{m}^{-1}$ as seen in Fig. 3(b). Shifting shock II toward the end of the PWFA reduces the acceleration length such that, just before the start of the gas jet density downramp, the maximum witness energy reduces to $17 \pm 1 \mathrm{MeV}$, barely above the low energy background. This confirms shock II as the source of witness injection. In the absence of other injection mechanisms and unique for the scenario with the shock wire in place, the injection of the witness is solely attributed to density downramp injection.

Additionally, tuning the accelerating gradient, autonomous from the acceleration length, is possible by adjusting the plasma density and expected to scale as $E_{z} \propto \sqrt{n_{p}}[40,56]$. To investigate this, the wire is placed at the jet center, fixing the point of injection and thus acceleration length. Scaling plasma density from $3.0 \times 10^{18} \mathrm{~cm}^{-3}$ up to $8.2 \times 10^{18} \mathrm{~cm}^{-3}$, we tune the acceleration gradient manifesting in higher witness energies following the expected scaling, as is shown in Fig. 4. At a plasma density of $8.2 \times 10^{18} \mathrm{~cm}^{-3}$ a maximum acceleration gradient of $121 \mathrm{GV} \mathrm{m}^{-1}$ is achieved.

In summary, making use of a high peak-current driver originating from a preceding LWFA, we have demonstrated the possibility of DDI in PWFAs at gradual ramps, as advised for high quality beam injection. These results provide a promising outlook for oncoming commissioning experiments at conventional sources, e.g., FACET-II and the intention to utilize their upgraded driver peak current to inject at gradual downramps where the wavelength of the wake adiabatically increases [26]. This concept can then be scaled at conventional accelerators to much higher energies and repetition rates, connecting a broad community striving toward the generation of energetic, ultrashort, and ultralow emittance beams. Meanwhile, compact LPWFAs' short beam durations of $\sim 15$ fs allows operation of PWFAs in a high-density regime that is hardly accessible for conventional electron sources, yielding increased accelerating gradients while opening a pathway toward ultrahigh-brightness beam production [36]. The next step for bunch quality improvement is to tune properties of the density transition, which our setup lacks at this point. Properly finetuning the density step promises to reduce energy spread and emittance [17]. Witness bunch creation with only few tens nm emittance is considered feasible [18,19]. The presented DDI 
(a) $\mathrm{dQ} / \mathrm{d} E / \mathrm{d} \theta[\mathrm{pC} / \mathrm{MeV} / \mathrm{mrad}]$

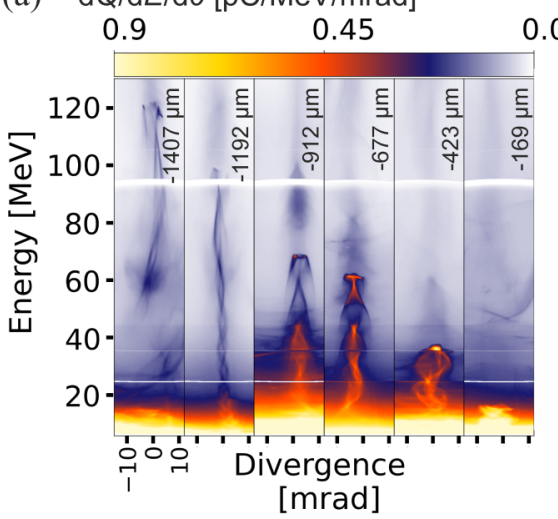

(b)

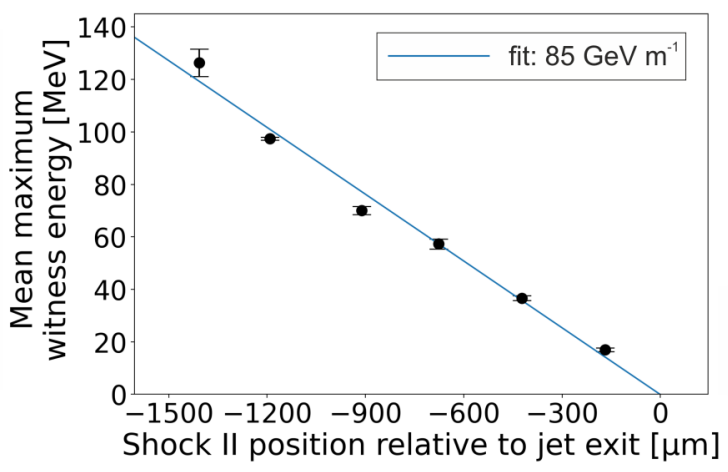

FIG. 3. Witness energy distribution for different positions of injection in the PWFA, operating at a plateau plasma density of $4.0 \times$ $10^{18} \mathrm{~cm}^{-3}$. Panel (a) shows representative shots for different wire positions along the jet. The inset numbers show the positions of shock II relative to the jet exit as determined by probe images. The reference position with the wire at the jet center places shock II $\sim 0.7 \mathrm{~mm}$ upstream from the jet exit. (b) Mean witness maximum peak energy dependence on acceleration length. Error bars represent the S.E.M., and each set consists of 2 to 10 shots.

at a previously inaccessible regime of plasma densities above $10^{18} \mathrm{~cm}^{-3}$, utilizing drivers with peak-currents beyond $10 \mathrm{kA}$, represents an essential step toward exploring PWFAs, both conventional or LWFA driven, as compact sources for high brightness [36] and ultrafast [57,58] science.

\section{ACKNOWLEDGMENTS}

This project was fully supported by the Helmholtz association under program Matter and Technology, topic Accelerator Research and Development. The authors gratefully acknowledge the GWK support for funding this project by providing computing time through the Center for Information Services and HPC (ZIH) at TU Dresden on the HRSK-II. This work was partially funded by the Center of Advanced Systems Understanding (CASUS) which is financed by Germany's Federal Ministry of Education and Research (BMBF) and by the Saxon Ministry for Science, Culture and Tourism (SMWK) with tax funds on the basis of the budget approved by the Saxon State Parliament. S.C., O.K., G.R., and A.Kn. were supported by the European Research Council (ERC) under the European Union's Horizon 2020 research and innovation programme (Miniature beam-driven Plasma Accelerators project, ERC Grant Agreement No. 715807). F.M.F. was funded by the Max-Planck School of Photonics, F.H. is funded by the BMBF through the Verbundforschungsprojekt "Ultrafast Imaging of Plasmas (ULFI)", A.Dö. and S.K. are funded by the Centre for Advanced Laser Applications (CALA). B.H., T.H., and A.N. were supported by the European Research Concil (ERC) under the European Union's Horizon 2020 research and innovation programme (NeXource, ERC Grant Agreement No. 865877) and by the Science and Technology Facilities Council (STFC) ST/S006214/1 PWFA-FEL.

\section{APPENDIX}

\section{Influence of the wire on the bunch evolution}

The plasma density and its profile play a critical role in recapturing the drive beam at the second jet. For this reason,
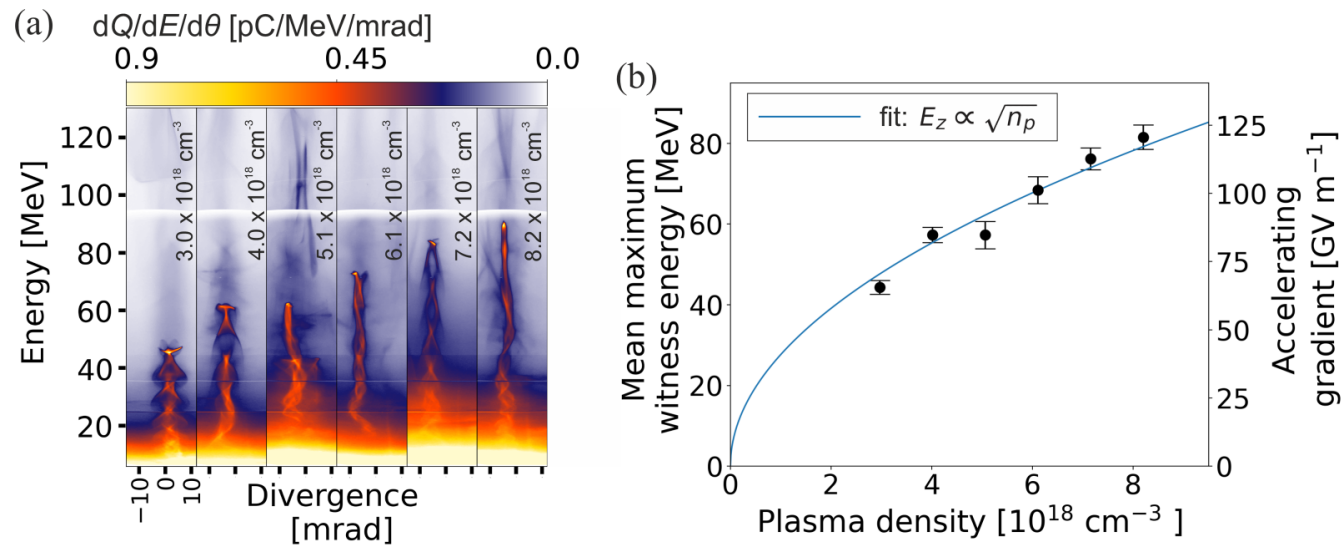

FIG. 4. Witness energy dependency on plasma density. Panel (a) shows representative shots with the PWFA operating at densities between $3.0 \times 10^{18} \mathrm{~cm}^{-3}$ and $8.2 \times 10^{18} \mathrm{~cm}^{-3}$ and the wire position fixed at the center of the PWFA. Panel (b) shows the energy dependency on plasma density. The left axis shows maximum witness energy with the data points representing the measured mean energy for sets consisting of 8 to 10 shots and the error bars depicting the S.E.M. The right axis shows the acceleration gradient. 

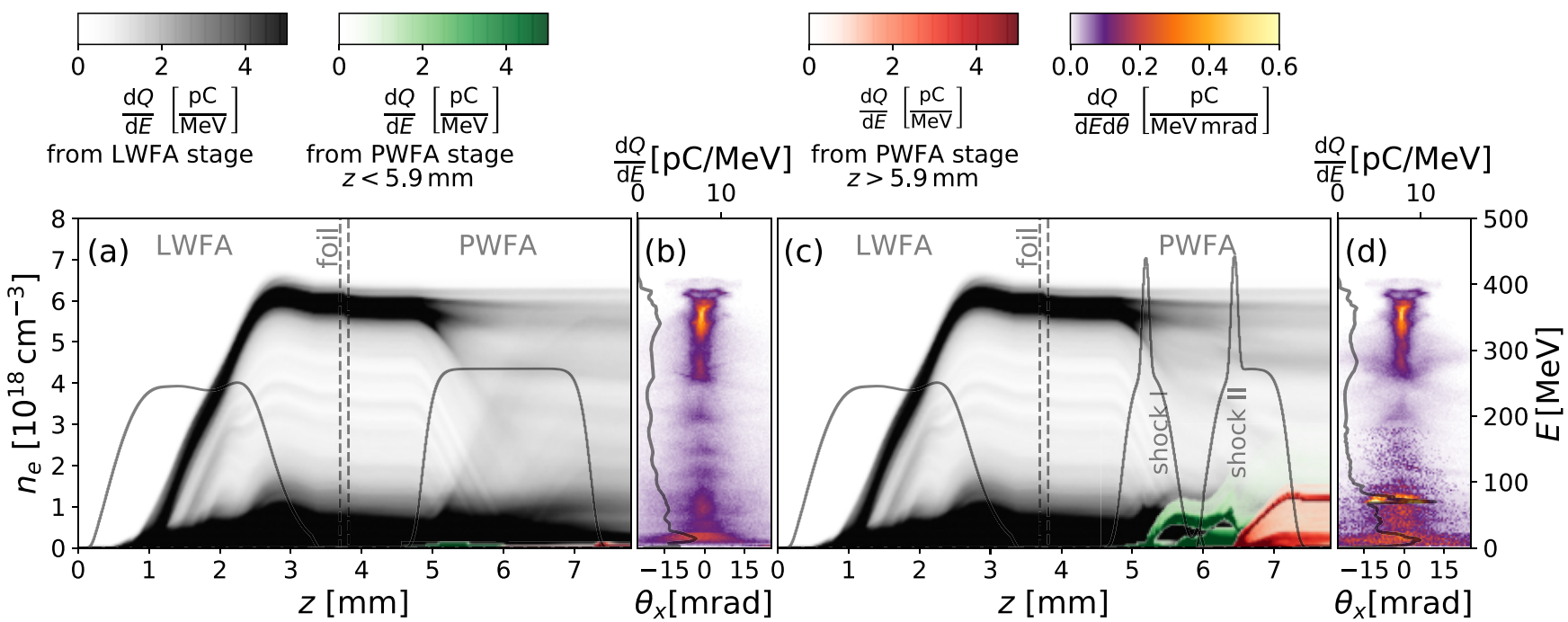

FIG. 5. Direct comparison of setup (simulated) with and without wire in place.

our experiment was explicitly designed not to significantly influence the up-ramp region of the gas-jet, where the beam capturing and refocusing occurs, on which we will detail in the next section. This is achieved by using a wire, which creates only a very localized disruption in the gas-flow, without significantly influencing the remainder of the gas-jet. This is contrary to e.g. a razor blade, which would completely remove the front section of the gas jet, which is required for beam capturing.

A simulation of the plasma lensing in absence of a wire, with a direct comparison to the simulation with shocks created by the wire (as included in the manuscript), is given in Fig. 5. The synthetic spectrometer data [Figs. 5(b) and 5(d)] show very similar bunch structures. In agreement with experimental measurements, the driver's FWHM charge are $Q_{\mathrm{FWHM}}=$ $44 \mathrm{pC}$ for the double-shock case and $Q_{\mathrm{FWHM}}=47 \mathrm{pC}$ for the unperturbed scenario.

\section{Details on beam recapture}

Mentioned in the main manuscript, the initial part of the hydrogen jet acts as a passive plasma lens to refocus a substantial part of the driver beam. A part of the driver beam is not refocused and thus excluded from the measured FWHM divergence value in the experiment.

In order to illustrate the effect of this selection in the experiment, we analyzed the bunch divergence as the bunch propagates through the PWFA stage using a PIC simulation (Fig. 6).

The strength of the focusing effect via plasma lensing depends on the radial (and longitudinal) position of electrons in the bunch.

Before plasma lensing occurs, $80 \%$ of the bunch charge is located within the FWHM divergence region (enclosed by the solid black line in Fig. 6) which amounts to $\sim 12 \mathrm{mrad}$. After plasma lensing at the end of the PWFA stage, only $40 \%$ of the total bunch charge is within the FWHM. But the FWHM divergence has reduced by a factor two to $\sim 6 \mathrm{mrad}$.

\section{Details to Fig. 2(c)}

Figure 2(c) of the main text, separates the contributions to the final electrons distribution by origin, color-coded and overlayed in a single figure.

To depict the individual contributions to the final electron energy distribution more clearly, the separated contributions zoomed in to energies below $200 \mathrm{MeV}$ and the PWFA jet range only, are given in Fig. 7.

The driver beam [black in Fig. 2(c) in the main text] has a sizeable contribution to lower energies. This can partially be attributed to a secondary downramp injection at the LWFA gas jet exit, and partially due to deceleration of the main, higher energy fraction of the driver beam.

Electrons originating from the first shock [green in Fig. 2(c) in the manuscript] are initially accelerated at the low density region between both shocks. During the increase of plasma density toward the second shock, its main fraction is rephased to the dephasing region $(\chi \lesssim-2 \pi)$ and thus decelerated. The dephasing means these electrons are spatially separated from the electrons injected into the first cavity at the second shock.

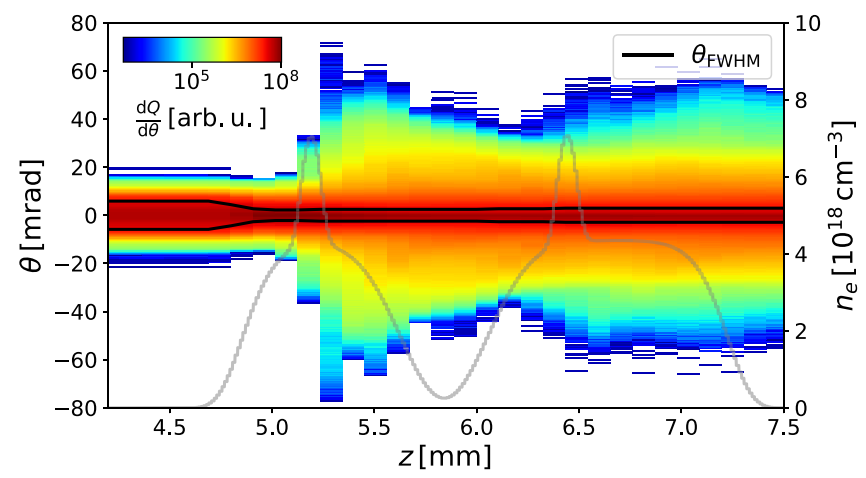

FIG. 6. Divergence evolution of the bunch while propagating through the PWFA stage. In black, the FWHM of the divergence is marked. When the bunch enters the PWFA stage and is being focused, the FWHM divergence $\theta_{\text {FWHM }}$ reduces. 


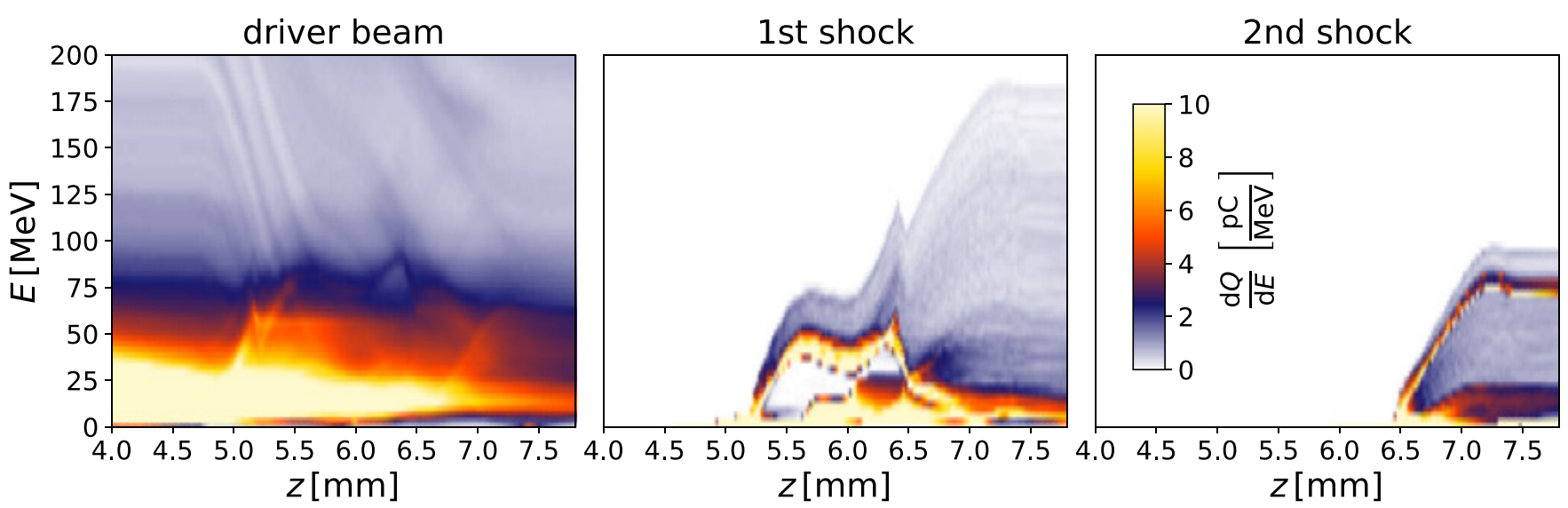

FIG. 7. Charge distribution evolution from simulation separated by electron origin.

Electrons originating from the second shock [red in Fig. 2(c) in the manuscript] are thus injected spatially separated and unperturbed by electrons from the first shock and distinguishable form the measured witness bunch around $74 \mathrm{MeV}$.
[1] P. Chen, J. M. Dawson, R. W. Huff, and T. Katsouleas, Acceleration of Electrons by the Interaction of a Bunched Electron Beam with a Plasma, Phys. Rev. Lett. 54, 693 (1985).

[2] S. Corde et al., High-field plasma acceleration in a highionization-potential gas, Nat. Commun. 7, 11898 (2016).

[3] K. V. Lotov, Blowout regimes of plasma wakefield acceleration, Phys. Rev. E 69, 046405 (2004).

[4] W. Lu, C. Huang, M. Zhou, W. B. Mori, and T. Katsouleas, Nonlinear Theory for Relativistic Plasma Wakefields in the Blowout Regime, Phys. Rev. Lett. 96, 165002 (2006).

[5] I. Blumenfeld, C. E. Clayton, F.-J. Decker, M. J. Hogan, C. Huang, R. Ischebeck, R. Iverson, C. Joshi, T. Katsouleas, N. Kirby et al., Energy doubling of $42 \mathrm{GeV}$ electrons in a metrescale plasma wakefield accelerator, Nature (London) 445, 741 (2007).

[6] M. Litos, E. Adli, W. An, C. I. Clarke, C. E. Clayton, S. Corde, J. P. Delahaye, R. J. England, A. S. Fisher, J. Frederico et al., High-efficiency acceleration of an electron beam in a plasma wakefield accelerator, Nature (London) 515, 92 (2014).

[7] A. Deng, O. S. Karger, T. Heinemann, A. Knetsch, P. Scherkl, G. G. Manahan, A. Beaton, D. Ullmann, G. Wittig, A. F. Habib et al., Generation and acceleration of electron bunches from a plasma photocathode, Nat. Phys. 15, 1156 (2019).

[8] B. W. J. McNeil and N. R. Thompson, X-ray free-electron lasers, Nat. Photonics 4, 814 (2010).

[9] E. Gschwendtner and P. Muggli, Plasma wakefield accelerators, Nat. Rev. Phys. 1, 246 (2019).

[10] H. Suk, N. Barov, J. B. Rosenzweig, and E. Esarey, Plasma Electron Trapping and Acceleration in a Plasma Wake Field Using a Density Transition, Phys. Rev. Lett. 86, 1011 (2001).

[11] G. Wittig, O. Karger, A. Knetsch, Y. Xi, A. Deng, J.B. Rosenzweig, D.L. Bruhwiler, J. Smith, G.G. Manahan, Z.M. Sheng, D.A. Jaroszynski, and B. Hidding, Optical plasma torch electron bunch generation in plasma wakefield accelerators, Phys. Rev. ST Accel. Beams 18, 081304 (2015).

[12] B. Hidding, G. Pretzler, J. B. Rosenzweig, T. Königstein, D. Schiller, and D. L. Bruhwiler, Ultracold Electron Bunch Gen- eration via Plasma Photocathode Emission and Acceleration in a Beam-Driven Plasma Blowout, Phys. Rev. Lett. 108, 035001 (2012).

[13] F. Li, J.F. Hua, X.L. Xu, C.J. Zhang, L.X. Yan, Y.C. Du, W.H. Huang, H.B. Chen, C.X. Tang et al., Generating High-Brightness Electron Beams via Ionization Injection by Transverse Colliding Lasers in a Plasma-Wakefield Accelerator, Phys. Rev. Lett. 111, 015003 (2013).

[14] Y. Wan, C. J. Zhang, F. Li, Y. P. Wu, J. F. Hua, C.-H. Pai, W. Lu, Y. Q. Gu, X. L. Xu, C. Joshi, and W. B. Mori, Colliding ionization injection in a plasma wakefield accelerator, Plasma Phys. Control. Fusion 58, 034015 (2016).

[15] E. Oz, S. Deng, T. Katsouleas, P. Muggli, C.D. Barnes, I. Blumenfeld, F. J. Decker, P. Emma, M. J. Hogan, R. Ischebeck et al., Ionization-Induced Electron Trapping in Ultrarelativistic Plasma Wakes, Phys. Rev. Lett. 98, 084801 (2007).

[16] A. Martinez de la Ossa, J. Grebenyuk, T. Mehrling, L. Schaper, and J. Osterhoff, High-Quality Electron Beams from BeamDriven Plasma Accelerators by Wakefield-Induced Ionization Injection, Phys. Rev. Lett. 111, 245003 (2013).

[17] A. Martinez de la Ossa, Z. Hu, M. J. V. Streeter, T. J. Mehrling, O. Kononenko, B. Sheeran, and J. Osterhoff, Optimizing density down-ramp injection for beam-driven plasma wakefield accelerators, Phys. Rev. Accel. 20, 091301 (2017).

[18] X. L. Xu, F. Li, W. An, T. N. Dalichaouch, P. Yu, W. Lu, C. Joshi, and W. B. Mori, High quality electron bunch generation using a longitudinal density-tailored plasma-based accelerator in the three-dimensional blowout regime, Phys. Rev. Acc. Beams 20, 111303 (2017).

[19] C. Zhang, C.-K. Huang, K. A. Marsh, X. L. Xu, F. Li, M. Hogan, V. Yakimenko, S. Corde, W. B. Mori, and C. Joshi, Effect of fluctuations in the down ramp plasma source profile on the emittance and current profile of the self-injected beam in a plasma wakefield accelerator, Phys. Rev. Acc. Beams 22, 111301 (2019).

[20] K. Schmid, A. Buck, C. M. S. Sears, J. M. Mikhailova, R. Tautz, D. Herrmann, M. Geissler, F. Krausz, and L. Veisz, 
Density-transition based electron injector for laser driven wakefield accelerators, Phys. Rev. ST Accel. Beams 13, 091301 (2010).

[21] A. Buck, J. Wenz, J. Xu, K. Khrennikov, K. Schmid, M. Heigoldt, J.M. Mikhailova, M. Geissler, B. Shen, F. Krausz, S. Karsch, and L. Veisz, Shock-Front Injector for High-Quality Laser-Plasma Acceleration, Phys. Rev. Lett. 110, 185006 (2013).

[22] M. Burza, A. Gonoskov, K. Svensson, F. Wojda, A. Persson, M. Hansson, G. Genoud, M. Marklund, C.-G. Wahlström, and O. Lundh, Laser wakefield acceleration using wire produced double density ramps, Phys. Rev. ST Accel. Beams 16, 011301 (2013).

[23] K.K. Swanson, H.E. Tsai, S.K. Barber, R. Lehe, H.S. Mao, S. Steinke, J. vanTilborg, K. Nakamura, C.G.R. Geddes, C.B. Schroeder, E. Esarey, and W.P. Leemans, Control of tunable, monoenergetic laser-plasma-accelerated electron beams using a shock-induced density downramp injector, Phys. Rev. ST Accel. Beams 20, 051301 (2017).

[24] S. K. Barber, J. vanTilborg, C. B. Schroeder, R. Lehe, H. E. Tsai, K. K. Swanson, S. Steinke, K. Nakamura, C. G. R. Geddes, C. Benedetti, E. Esarey, and W. P. Leemans, Measured Emittance Dependence on the Injection Method in Laser Plasma Accelerators, Phys. Rev. Lett. 119, 104801 (2017).

[25] H.-E. Tsai, K. K. Swanson, S. K. Barber, R. Lehe, H.-S. Mao, D. E. Mittelberger, S. Steinke, K. Nakamura, J. van Tilborg, C. Schroeder et al., Control of quasi-monoenergetic electron beams from laser-plasma accelerators with adjustable shock density profile, Phys. Plasmas 25, 043107 (2018).

[26] C. Joshi, E. Adli, W. An, C. E. Clayton, S. Corde, S. Gessner, M. J. Hogan, M. Litos, W. Lu, K. A. Marsh et al., Plasma wakefield acceleration experiments at FACET II, Plasma Phys. Control. Fusion 60, 034001 (2018).

[27] A. Aschikhin, C. Behrens, S. Bohlen, J. Dale, N. Delbos, L. di Lucchio, E. Elsen, J.-H. Erbe, M. Felber, B. Foster et al., The FLASHForward facility at DESY, Nucl. Instrum. Methods Phys. Res. A 806, 175 (2016).

[28] A. Knetsch, B. Sheeran, L. Boulton, P. Niknejadi, K. Põder, L. Schaper, M. Zeng, S. Bohlen, G. Boyle, T. Brümmer et al., Controlled density-downramp injection in a beam-driven plasma wakefield accelerator, Phys. Rev. Accel. Beams 24, 101302 (2020).

[29] D. Ullmann, P. Scherkl, A. Knetsch, T. Heinemann, A. Sutherland, A. F. Habib, O. S. Karger, A. Beaton, G. G. Manahan, A. Deng et al., All-optical density downramp injection in electron-driven plasma wakefield accelerators, arXiv:2007.12634 [physics.plasm-ph] (2020).

[30] T. Kurz, T. Heinemann, M. F. Gilljohann, Y. Y. Chang, J. P. Couperus Cabadağ, A. Debus, O. Kononenko, R. Pausch, S. Schöbel, R. W. Assmann et al., Demonstration of a compact plasma accelerator powered by laser-accelerated electron beams, Nat. Commun. 12, 2895 (2021).

[31] B. Hidding, T. Königstein, J. Osterholz, S. Karsch, O. Willi, and G. Pretzler, Monoenergetic Energy Doubling in a Hybrid LaserPlasma Wakefield Accelerator, Phys. Rev. Lett. 104, 195002 (2010).

[32] A. Martinez de la Ossa, R. W. Assmann, M. Bussmann, S. Corde, J. P. Couperus Cabadağ, A. Debus, A. Döpp, A. Ferran Pousa, M. F. Gilljohann, T. Heinemann et al., Hybrid LWFAPWFA staging as a beam energy and brightness transformer: conceptual design and simulations, Philos. Trans. R. Soc. A 377, 20180175 (2019).

[33] J. P. Couperus, R. Pausch, A. Köhler, O. Zarini, J. M. Krmer, M. Garten, A. Huebl, R. Gebhardt, U. Helbig, S. Bock et al., Demonstration of a beam loaded nanocoulomb-class laser wakefield accelerator, Nat. Commun. 8, 487 (2017).

[34] Y. F. Li, D. Z. Li, K. Huang, M. Z. Tao, M. H. Li, J. R. Zhao, Y. Ma, X. Guo, J. G. Wang, M. Chen et al., Generation of $20 \mathrm{kA}$ electron beam from a laser wakefield accelerator, Phys. Plasmas 24, 023108 (2017).

[35] O. Lundh, J. Lim, C. Rechatin, L. Ammoura, A. Ben-Ismaïl, X Davoine, G. Gallot, J-P. Goddet, E. Lefebvre, V. Malka, and J. Faure, Few femtosecond, few kiloampere electron bunch produced by a laser-plasma accelerator, Nat. Phys. 7, 219 (2011).

[36] M. C. Thompson, J. B. Rosenzweig, and H. Suk, Plasma density transition trapping as a possible high-brightness electron beam source, Phys. Rev. ST Accel. Beams 7, 011301 (2004).

[37] U. Schramm, M. Bussmann, A. Irman, M. Siebold, K. Zeil, D. Albach, C. Bernert, S. Bock, F. Brack, J. Branco et al., First results with the novel petawatt laser acceleration facility in Dresden, J. Phys.: Conf. Ser. 874, 012028 (2017).

[38] A. Irman, J. P. Couperus, A. Debus, A. Köhler, J. M. Krämer, R. Pausch, O. Zarini, and U. Schramm, Improved performance of laser wakefield acceleration by tailored self-truncated ionization injection, Plasma Phys. Control. Fusion 60, 044015 (2018).

[39] O. Zarini, A. Irman, A. Debus, A. Köhler, J. P. Couperus, R. Pausch, T. Kurz, S. Schobel, H. Meissner, M. Bussmann, and U. Schramm, Advanced methods for temporal reconstruction of modulated electron bunches, in 2018 IEEE Advanced Accelerator Concepts Workshop (AAC) (IEEE, 2018), pp. 1-5.

[40] M. C. Downer, R. Zgadzaj, A. Debus, U. Schramm, and M. C. Kaluza, Diagnostics for plasma-based electron accelerators, Rev. Mod. Phys. 90, 035002 (2018).

[41] O. Zarini, J. P. Couperus Cabadağ, Y.-Y. Chang, A. Köhler, T. Kurz, S. Schöbel, W. Seidel, M. Bussmann, U. Schramm, and A. Debus, Multi-octave high-dynamic range optical spectrometer for single-pulse diagnostic applications, arXiv:2104.08539 (2021).

[42] G. Raj, O. Kononenko, M.F. Gilljohann, A. Doche, X. Davoine, C. Caizergues, Y.Y. Chang, J.P. CouperusCabadağ, A. Debus, H. Ding, M. Forster, J.P. Goddet, T. Heinemann, T. Kluge, T. Kurz, R. Pausch, P. Rousseau, P. SanMiguelClaveria, S. Schobel, A. Siciak et al., Probing ultrafast magnetic-field generation by current filamentation instability in femtosecond relativistic laser-matter interactions, Phys. Rev. Research 2, 023123 (2020).

[43] M. F. Gilljohann, H. Ding, A. Dopp, J. Gotzfried, S. Schindler, G. Schilling, S. Corde, A. Debus, T. Heinemann, B. Hidding, S.M. Hooker, A. Irman, O. Kononenko, T. Kurz, A. MartinezdelaOssa, U. Schramm, and S. Karsch, Direct Observation of Plasma Waves and Dynamics Induced by LaserAccelerated Electron Beams, Phys. Rev. X 9, 011046 (2019).

[44] C.E. Clayton, B.E. Blue, E.S. Dodd, C. Joshi, K.A. Marsh, W.B. Mori, S. Wang, P. Catravas, S. Chattopadhyay, E. Esarey, W.P. Leemans, R. Assmann, F.J. Decker, M.J. Hogan, R. Iverson, P. Raimondi, R.H. Siemann, D. Walz, T. Katsouleas, S. Lee et al., Transverse Envelope Dynamics of a 28.5-GeV Electron Beam in a Long Plasma, Phys. Rev. Lett. 88, 154801 (2002).

[45] S. Kuschel, D. Hollatz, T. Heinemann, O. Karger, M. B. Schwab, D. Ullmann, A. Knetsch, A. Seidel, C. Rodel, M. 
Yeung, M. Leier, A. Blinne, H. Ding, T. Kurz, D. J. Corvan, A. Savert, S. Karsch, M.C. Kaluza, B. Hidding, and M. Zepf, Demonstration of passive plasma lensing of a laser wakefield accelerated electron bunch, Phys. Rev. ST Accel. Beams 19, 071301 (2016).

[46] C.E. Doss, E. Adli, R. Ariniello, J. Cary, S. Corde, B. Hidding, M.J. Hogan, K. Hunt-Stone, C. Joshi, K.A. Marsh, J.B. Rosenzweig, N. Vafaei-Najafabadi, V. Yakimenko, and M. Litos, Laser-ionized, beam-driven, underdense, passive thin plasma lens, Phys. Rev. ST Accel. Beams 22, 111001 (2019).

[47] R. Ariniello, C. E. Doss, K. Hunt-Stone, J. R. Cary, and M. D. Litos, Transverse beam dynamics in a plasma density ramp, Phys. Rev. ST Accel. Beams 22, 041304 (2019).

[48] M. Bussmann et al., Radiative signatures of the relativistic Kelvin-Helmholtz instability, in Proceedings of the International Conference on High Performance Computing, Networking, Storage and Analysis (ACM, New York, NY, 2013), pp. $1-12$.

[49] J. P. Couperus, A. Köhler, T. Wolterink, A. Jochmann, O. Zarini, H. Bastiaens, K. Boller, A. Irman, and U. Schramm, Tomographic characterisation of gas-jet targets for laser wakefield acceleration, Nucl. Instrum. Methods Phys. Res. A 830, 504 (2016).

[50] D. Bauer and P. Mulser, Exact field ionization rates in the barrier-suppression regime from numerical time-dependent Schrödinger-equation calculations, Phys. Rev. A 59, 569 (1999).

[51] N. B. Delone and V. P. Krainov, Tunneling and barrier- suppression ionization of atoms and ions in a laser radiation field, Phys. Usp. 41, 469 (1998).

[52] R. Lehe, A. Lifschitz, C. Thaury, V. Malka, and X. Davoine, Numerical growth of emittance in simulations of laserwakefield acceleration, Phys. Rev. ST Accel. Beams 16, 021301 (2013).

[53] J. P. Boris, Relativistic plasma simulation-Optimization of a hybrid code, in Proc. 4th Conf. on Num. Sim. of Plasmas, ADA023511 (Defense Technical Information Center, Naval Research Laboratory, Washington, D.C., 1970), pp. 3-67.

[54] T. Z. Esirkepov, Exact charge conservation scheme for particlein-cell simulation with an arbitrary form-factor, Comput. Phys. Commun. 135, 144 (2001).

[55] R. Pausch, J. P. Couperus Cabadağ, S. Bastrakov, M. Bussmann, A. Irman, T. Kurz, S. Schöbel, U. Schramm, K. Steiniger, P. Ufer et al., PIConGPU setup: LPWFA downramp injection (2021), available at https://rodare.hzdr.de/record/840.

[56] W. Lu, C. Huang, M. Zhou, M. Tzoufras, F. S. Tsung, W. B. Mori, and T. Katsouleas, A nonlinear theory for multidimensional relativistic plasma wave wakefields, Phys. Plasmas $\mathbf{1 3}$ 056709 (2006).

[57] B. M. Alotaibi, R. Altuijri, A. F. Habib, A. Hala, B. Hidding, S. M. Khalil, B. W. J. McNeil, and P. Traczykowski, Plasma wakefield accelerator driven coherent spontaneous emission from an energy chirped electron pulse, New J. Phys. 22, 013037 (2020).

[58] C. Emma, X. Xu, A. Fisher, J. P. MacArthur, J. Cryan, M. J. Hogan, P. Musumeci, G. White, and A. Marinelli, Terawatt attosecond X-ray source driven by a plasma accelerator, APL Photonics 6, 076107 (2021). 